\title{
COMPOSING FUNCTIONS OF BOUNDED VARIATION
}

\author{
MICHAEL JOSEPHY
}

ABstract. We find necessary and sufficient conditions on $g$ such that $f \circ g$ (resp. $g \circ f$ ) is a function of bounded variation for all $f$ of bounded variation.

Let $\mathbf{I}=[0,1]$, and let $\mathbf{B V}$ denote the set of functions $f: \mathbf{I} \rightarrow \mathbf{I}$ of bounded variation. BV is not closed under composition (example: $f \circ g$ where $f(x)=\sqrt{x}$; $g(0)=0, g(x)=x^{2} \sin ^{2}(1 / x)$ for $\left.x \neq 0\right)$. Here we characterize those functions $g$ whose right (resp. left) composition operation $f \rightarrow f \circ g$ (resp. $f \rightarrow g \circ f$ ) preserves BV.

If $f: \mathbf{I} \rightarrow \mathbf{R}$, define $v(f, a, b)$ as the variation of $f$ on the subinterval $[a, b]$, and $v(f)=v(f, 0,1)$. For a positive integer $N$, let $J_{N}=\{X \subseteq I: X$ can be expressed as a union of $N$ intervals (where the intervals may be open or closed at either end and we allow singletons as degenerate closed intervals). Since any interval is a union of two subintervals, we see $J_{N} \subset J_{N+1}$. A function $f: \mathbf{I} \rightarrow \mathbf{R}$ is said to be of $N$-bounded variation if $f^{-1}([a, b]) \in J_{N}$ for all $[a, b] \subseteq \mathbf{R}$. Let $\mathbf{B V}(N)$ be the set of all functions $f: \mathbf{I} \rightarrow \mathbf{I}$ of $N$-bounded variation, and $\mathbf{B V}^{\prime}(N)$ the set of all bounded functions $f: \mathbf{I} \rightarrow \mathbf{R}$ of $N$-bounded variation.

LEMMA 1. Every function in $\mathbf{B V}^{\prime}(N)$ is of bounded variation.

Proof. Suppose $h \in \mathbf{B V}^{\prime}(N)$ and $|h(x)|<M$ for all $x \in \mathbf{I}$. We show $v(h)<$ $4 M(N+1)$. If $v(h)>4 M(N+1)$, then there exists a partition $\left\{x_{0}, \ldots, x_{n}\right\}$ of I with

$$
\sum_{i=1}^{n}\left|h\left(x_{i}\right)-h\left(x_{i-1}\right)\right|>4 M(N+1)
$$

Some $\left[a^{\prime}, b^{\prime}\right] \subseteq[-M, M]$ with $a^{\prime}<b^{\prime}$ is covered more than $2(N+1)$ times by intervals $\left[h\left(x_{i-1}\right), h\left(x_{i}\right)\right]$ or $\left[h\left(x_{i}\right), h\left(x_{i-1}\right)\right]$. At least $N+1$ are of the type $\left[h\left(x_{i-1}\right), h\left(x_{i}\right)\right]$, say, so

$$
\left[a^{\prime}, b^{\prime}\right] \subseteq \bigcap_{j=1}^{N+1}\left[h\left(y_{j}\right), h\left(z_{j}\right)\right]
$$

where each $\left(y_{j}, z_{j}\right)=\left(x_{i-1}, x_{i}\right)$ for some $i$, and $y_{1}<z_{1}<\cdots<y_{N+1}<z_{N+1}$. Then $h\left(y_{j}\right) \leqslant a^{\prime}<b^{\prime} \leqslant h\left(z_{j}\right)$ for all $j$. Take $a$ between $a^{\prime}$ and $b^{\prime}$; take $b$ greater than

Received by the editors March 1, 1980 and, in revised form, October 20, 1980.

1980 Mathematics Subject Classification. Primary 26A45; Secondary 04A05, 26A16.

Key words and phrases. Function of bounded variation, composition, Lipschitz condition. 
every $h\left(z_{j}\right)$. Then $h\left(y_{j}\right) \notin[a, b], h\left(z_{j}\right) \in[a, b]$ for all $j$. Hence $h^{-1}([a, b])$ is union of no fewer than $N+1$ intervals, and so is not in $J_{N}$, a contradiction. $\therefore h$ is of bounded variation.

LEMMA 2. Let $h_{i}: \mathbf{I} \rightarrow \mathbf{I}, i=1,2, \ldots$, be functions which assume only the values 0 and $3^{-i}$. If $h=\sum_{i=1}^{\infty} h_{i}$, then $v(h)>\frac{1}{6} \sum_{i=1}^{\infty} v\left(h_{i}\right)$. In particular, if some $h_{i} \notin \mathrm{BV}$, then $h \notin \mathrm{BV}$.

Proof. Let $p$ be a discontinuity of $h_{i}$ which is not a discontinuity of $h_{j}$ for $j<i$. Then $p$ is a discontinuity of $h$, contributing a jump of at least $3^{-i}-\sum_{j>i} 3^{-j}=\frac{1}{2} 3^{-i}$ in $h$, but contributing no more than $2 \sum_{j>i} 3^{-j}=3^{-i+1}$ to $\sum v\left(h_{i}\right)$. If each $h_{i} \in \mathrm{BV}$, i.e. has only a finite number of discontinuities, the inequality follows. Otherwise some $h_{i}$ has infinitely many discontinuities, and $v(h)=v\left(h_{i}\right)=\infty$.

THEOREM 3. For $g: \mathbf{I} \rightarrow \mathbf{I}$, the composition $f \circ g$ belongs to $\mathbf{B V}$ for all $f$ in $\mathbf{B V}$ iff $g \in \mathbf{B V}(N)$ for some $N$.

Proof. Sufficiency. Suppose $f: \mathbf{I} \rightarrow \mathbf{R}$ is increasing, and $g \in \mathbf{B V}(N)$. Then $f \circ g$ $\in \mathbf{B V}^{\prime}(N)$ because $f^{-1}([a, b])=\left[f^{-1}(a), f^{-1}(b)\right]$, and hence $(f \circ g)^{-1}([a, b]) \in J_{N}$ for all $a, b$. Moreover, any $f \in \mathbf{B V}$ can be expressed as a difference of two increasing functions $f=f_{1}-f_{2}$, and since $f_{1} \circ g, f_{2} \circ g \in \mathbf{B V}^{\prime}(N)$, we have $f \circ g=f_{1} \circ g-$ $f_{2} \circ g \in \mathbf{B V}$, using Lemma 1 .

Necessity. Suppose for all $N, g \notin \mathbf{B V}(N)$. Find intervals $I_{n} \subseteq \mathbf{I}, n=1,2, \ldots$, such that $g^{-1}\left(I_{n}\right) \notin J_{3^{n}}$, i.e. $g^{-1}\left(I_{n}\right)$ cannot be expressed as a union of $3^{n}$ intervals. Define $f_{n}=3^{-n} \chi_{I_{n}}$, and $f=\sum_{n=1}^{\infty} f_{n}$. Since $v\left(f_{n}\right)<2 \cdot 3^{-n}$, we have $v(f)<\sum v\left(f_{n}\right)$ $<2 \sum 3^{-n}<\infty$, so $f \in \mathbf{B V}$. On the other hand, $f_{n} \circ g=3^{-n} \chi_{g^{-1}\left(I_{n}\right)}$, so $v\left(f_{n} \circ g\right)$ $\geqslant 2 \cdot 3^{-n} \cdot 3^{n}=2$. By Lemma $2, v(f \circ g)=\infty$, i.e. $f \circ g \notin \mathbf{B V}$.

TheOREM 4. For $g: \mathbf{I} \rightarrow \mathbf{I}$, the composition $g \circ f$ belongs to $\mathbf{B V}$ for all $f$ in $\mathbf{B V}$ iff $g$ satisfies a Lipschitz condition on $\mathbf{I}$.

Proof. Sufficiency. Suppose $|g(y)-g(z)|<M|y-z|$ for all $y, z \in \mathbf{I}$, and suppose $f \in \mathbf{B V}$ with $v(f)=L$. If $P=\left\{x_{0}, \ldots, x_{n}\right\}$ is a partition of $\mathbf{I}$,

$$
\sum_{P}\left|\Delta(g \circ f)_{i}\right| \leqslant \sum_{i=1}^{n} M\left|f\left(x_{i}\right)-f\left(x_{i-1}\right)\right|<L M,
$$

so $g \circ f \in \mathbf{B V}$.

Necessity. If $g$ does not satisfy a Lipschitz condition on $\mathbf{I}$, there exist $y_{n}^{\prime \prime}<z_{n}^{\prime \prime}$ with $\left|g\left(y_{n}^{\prime \prime}\right)-g\left(z_{n}^{\prime \prime}\right)\right|>\left(n^{2}+n\right)\left|y_{n}^{\prime \prime}-z_{n}^{\prime \prime}\right|$ for any $n=1,2, \ldots$ Since I is compact, there is a convergent subsequence $\left\{y_{n}^{\prime}\right\}$ of $\left\{y_{n}^{\prime \prime}\right\}$. Say $y_{n}^{\prime} \rightarrow y$. Take a further subsequence $\left\{y_{n}\right\}$ with $\left|y-y_{n}\right|<(n+1)^{-2}$. Take $\left\{z_{n}\right\}$ to be the corresponding subsequence of $\left\{z_{n}^{\prime \prime}\right\}$, and $\delta_{n}=\left|y_{n}-z_{n}\right|$. We have $\left|g\left(y_{n}\right)-g\left(z_{n}\right)\right|>\left(n^{2}+n\right) \delta_{n}$, and hence $\delta_{n}<\left(n^{2}+n\right)^{-1}$.

Define $f$ by $f(0)=0, f(1)=y_{1}$, while on $\left[(n+1)^{-1}, n^{-1}\right)$,

$$
f(x)= \begin{cases}y_{n} & \text { if } x-(n+1)^{-1} \text { is a multiple of } \delta_{n}, \\ z_{n} & \text { otherwise. }\end{cases}
$$


For a fixed $n$, let $m^{\prime}=\delta_{n}^{-1}\left(n^{2}+n\right)^{-1}$, and $m=\max \left\{k \in \mathbf{Z}: k<m^{\prime}\right\}$. Since $m^{\prime}>$ 1, we have $m^{\prime} / 2<m<m^{\prime}$. Consider the partition $P$ of $\left[(n+1)^{-1}, n^{-1}\right]$ :

$$
P=\left\{(n+1)^{-1}+\frac{1}{2} r \delta_{n}: r=0,1, \ldots, 2 m\right\} \cup\left\{n^{-1}\right\} \text {. }
$$

Then

$$
\sum_{P}\left|\Delta(g \circ f)_{i}\right| \geqslant 2 m\left|g\left(y_{n}\right)-g\left(z_{n}\right)\right|>m^{\prime}\left(n^{2}+n\right) \delta_{n}=1,
$$

so $v\left(g \circ f,(n+1)^{-1}, n^{-1}\right)>1$ : whence $g \circ f \notin \mathbf{B V}$.

On the other hand, since $f$ is a step function on $\left[(n+1)^{-1}, n^{-1}\right]$,

$$
\begin{aligned}
v\left(f,(n+1)^{-1}, n^{-1}\right) & =2 m\left|y_{n}-z_{n}\right|+\left|y_{n-1}-t_{n}\right| \quad \text { where } t_{n}=y_{n} \text { or } z_{n} \\
& <2 m^{\prime} \delta_{n}+\left|y_{n-1}-y\right|+\left|y-y_{n}\right|+\left|y_{n}-z_{n}\right| \\
& <2\left(n^{2}+n\right)^{-1}+n^{-2}+(n+1)^{-2}+n^{-2}<5 n^{-2} .
\end{aligned}
$$

Since $\Sigma n^{-2}$ converges, $f \in \mathbf{B V}$.

Chaika and Waterman [1] obtained a theorem analogous to our Theorem 4, but for certain classes larger than BV. Interestingly, they found the Lipschitz condition to be necessary and sufficient in those cases as well.

\section{REFERENCES}

1. M. Chaika and D. Waterman, On the invariance of certain classes of functions under compasition, Proc. Amer. Math. Soc. 43 (1974), 345-348. MR 48 \#8704.

Escuela de Matemática, Universidad de Costa Rica, San José, Costa Rica 